\title{
Application of the control volume method to a mathematical model of cell migration
}

\author{
D. G. Mallet I. W. Turner G. J. Pettet*
}

(Received 8 August 2003; revised 29 January 2004)

\begin{abstract}
In recent years, mathematical models of cell migration have become increasingly complex. These models have evolved from simple diffusion models to computationally troublesome reaction-diffusionadvection models. As such, the use of "black box" numerical solvers has become less appropriate. We discuss the application of the control volume technique for resolving a complicated nonlinear cell migration model. The nonlinearity is treated using an inexact Newton solver and flux limiting ensures that the cell migration fronts are captured adequately. Specifically, we analyse the model due to Perumpanani et al. (1999), comparing the numerical results of the proposed computational model developed in this research to previous results published by other researchers. We show that the finite volume computational model captures the physics of the processes with good accuracy using coarse meshes.
\end{abstract}

*School of Mathematical Sciences, Queensland University of Technology, Brisbane, Australia. mailto:dg.mallet@qut.edu.au

See http://anziamj.austms.org.au/V45/CTAC2003/Mall/home.html for this article, (c) Austral. Mathematical Soc. 2004. Published August 15, 2004. ISSN 1446-8735 


\section{Contents}

1 Introduction

C892

2 The Perumpanani et al. model $\quad$ C893

2.1 Discretisation . . . . . . . . . . . . . . . . C894

2.2 Approximation of the flux term at the CV faces . . . . . C895

2.3 Nonlinear solution method . . . . . . . . . . . . C897

3 Results and comparisons

C897

4 Conclusions

C902

References

C903

\section{Introduction}

Cell migration is an important process in such phenomena as tumour invasion, wound healing and tissue generation. Of particular interest here is haptotaxis - that subset of cellular migration that occurs when cells migrate in response to a gradient in extracellular matrix (ECM) density. This is intended to be representative of the density of sites in the ECM to which a migrating cell may be able to attach. The cells attach to the matrix, a collection of proteins and polysaccharides secreted by cells and organized into a mesh [1], and consequently migrate in the direction of increasing concentration of ECM. In this work we examine the numerical solution of the mathematical model due to Perumpanani et al. [9], which was proposed to describe this process.

The Perumpanani et al. model involves two partial differential equations (PDEs) for the conservation of invasive tumour cell density and ECM density. Due to the complex, coupled nature and nonlinearity of the model, analyti- 
cal solutions are not able to be found. Typically, the Numerical Algorithms Group (NAG) routine D03PCF [6] has been utilised [2, 7, 8, e.g.] in the solution of models such as that proposed by Perumpanani et al. [9]. Unfortunately the usefulness of this NAG routine, which has been designed for parabolic equations, is limited due to the mixed parabolic-hyperbolic nature of cell migration models and the existence of sharp fronts and spikes in their solutions. Often these routines must be utilised with extremely fine meshes, resulting in high computational overheads, in order to obtain numerical results that are of any credibility. These limitations have led to studies of the LaxFriedrichs, Lax-Wendroff and Kurganov-Tadmor schemes in relation to the Perumpanani et al. model $[4,5]$. Here, we have used a vertex-centred control volume scheme to provide accurate numerical solutions to a cell migration model. We investigate various temporal and spatial weighting schemes and their effectiveness in producing accurate solution behaviour.

In the section to follow we outline the mathematical model to be studied, and the corresponding computational model. Results and comparisons are presented in Section 3, followed by conclusions and a discussion of possibilities for future research in Section 4.

\section{The Perumpanani et al. model}

The dimensionless cell-haptotaxis model of Perumpanani et al. [9] considers the evolution over time of malignant cells, denoted $u(x, t)$, and connective tissue or ECM, denoted $c(x, t)$, to be governed by the model equations

$$
\begin{gathered}
u_{t}+\left(u c_{x}\right)_{x}-u(1-u)=0, \\
c_{t}+u c^{2}=0
\end{gathered}
$$

where subscript $t$ and $x$ represent partial differentiation with respect to time and space respectively. Furthermore, Neumann boundary conditions are im- 
posed at $x=0$ and $x=L$, and we choose initial conditions

$$
\begin{gathered}
u(x, 0)=0.5(1-\tanh (a x-b)), \\
c(x, 0)=c_{0}(1-u(x, 0)),
\end{gathered}
$$

where equation (3) reflects a step-like initial cell profile characterised by the arbitrary constant coefficients $a$ and $b$ that determine the steepness and position, respectively, of the initial cell front, and $c_{0}$ is the "normal" level of extracellular matrix before cell invasion. Throughout this work we take $a=$ 1.5 and $b=8$, indicating an initial cell population which has migrated over approximately $10 \%$ of the spatial domain, and $c_{0}=0.6$, a similar "normal" level of ECM [9].

Equation (2) relates ECM density changes over time to the interaction between cells and ECM. The invasive cell equation (1) relates changes in cell density to logistic proliferation of cells and the haptotactic migration of cells towards higher densities of ECM with a velocity proportional to the ECM density gradient. The initial conditions represent a situation where the cells are invading from left to right into a previously undisturbed region of ECM. Neumann boundary conditions are used to preserve the steady state cell and ECM levels and to ignore any external influences on the two species.

\subsection{Discretisation}

We discretise the model equations using the vertex centred control volume method with a uniform mesh (though it is possible to implement the method on a nonuniform mesh). Given the domain length $L$, the nodes $\left(x_{i}, i=\right.$ $1,2, \ldots, n)$ are placed uniformly along the $x$-axis between $x=0$ and $x=$ $L$. Control volumes are constructed around the nodes with boundaries at $x_{i+1 / 2}$ and $x_{i-1 / 2}$.

The discretisation proceeds by separately integrating equations (1) and (2) over an arbitrary control volume and subsequently applying the Mean Value 
Theorem, before integrating over time from $t_{n}$ to $t_{n+1}$ to give the discrete form of the equations:

$$
\begin{aligned}
F_{u_{i}} \equiv & \Delta x_{i}\left[u_{i}^{n+1}-u_{i}^{n}\right] \\
& +\delta t\left[\alpha\left(J_{i+1 / 2}^{n+1}-J_{i-1 / 2}^{n+1}\right)+(1-\alpha)\left(J_{i+1 / 2}^{n}-J_{i-1 / 2}^{n}\right)\right] \\
& -\delta t \alpha \Delta x_{i} u_{i}^{n+1}\left(1-u_{i}^{n+1}\right)-\delta t(1-\alpha) \Delta x_{i} u_{i}^{n}\left(1-u_{i}^{n}\right) \\
= & 0 \\
F_{c_{i}} \equiv & \Delta x_{i}\left[c_{i}^{n+1}-c_{i}^{n}\right]-\delta t \alpha \Delta x_{i} u_{i}^{n+1}\left(c_{i}^{n+1}\right)^{2}-\delta t(1-\alpha) \Delta x_{i} u_{i}^{n}\left(c_{i}^{n}\right)^{2} \\
= & 0
\end{aligned}
$$

where $\Delta x_{i}$ represents the control volume spacing, $\delta t$ is the time step, $\alpha$ is the temporal weighting coefficient and $J=u c_{x}$ represents the flux term of equation (1). Subscripts on $u$ and $c$ denote the spatial position in question (node points in the case of integer subscripts, CV faces otherwise), while superscripts denote the temporal position.

The temporal weighting coefficient allows for a fully explicit system with $\alpha=0$, a fully implicit system with $\alpha=1$, and a second order implicit Crank-Nicolson scheme with $\alpha=0.5$. In this work we disregard the explicit scheme due to the restrictions that it places on the spatial mesh size and temporal step size. While the fully implicit system is stable it is only first order accurate in time. On the other hand, the Crank-Nicolson scheme may produce non-monotonic results for certain time/spatial step combinations, but it is second order accurate in time.

\subsection{Approximation of the flux term at the $\mathrm{CV}$ faces}

In equation (5), approximations for the flux term $J$ at the $\mathrm{CV}$ faces are required. We consider three possible approximations for $J$, namely the first order upstream method, the second order averaging method, and the van Leer flux limiter. Regardless of which of these spatial weighting schemes we 
employ, the derivative in ECM is represented using the first order approximation

$$
\left[c_{x}\right]_{i+1 / 2}=\left(c_{i+1}-c_{i}\right) / \Delta x_{i+1},
$$

and the invasive cell density using the expression

$$
u_{i+1 / 2}=u_{i}+\sigma_{i+1 / 2}\left(u_{i+1}-u_{i}\right) / 2,
$$

where $\sigma_{i+1 / 2}$ is the flux limiter function, which depends on the chosen spatial weighting scheme and in the case of the van Leer flux limiter, also depends on the sensor $r$ according to the function $\sigma(r)=2 r /(1+r)$ [10]. The sensor is defined as the ratio of the cell fluxes at the upstream and second upstream $\mathrm{CV}$ faces, though other sensors, such as the ratio of ECM density gradients could also be employed.

In order to implement the Neumann boundary conditions we define the cell flux at both boundary $\mathrm{CV}$ faces to be identically zero.

As a result of the control volume discretisation we have two nonlinear equations, one for invasive cells (equation (5) with (7) and (8) used in the flux approximations) and one for connective tissue (equation (6)), defined at each of the $(n+1)$ node points across the spatial domain. Hence, in order to advance the solution in time, a system of $2(n+1)$ nonlinear equations of the form $\mathbf{F}(\mathbf{u})=\mathbf{0}$ must be solved at each time step for the unknown vector of solutions $\mathbf{u}$, which takes the block partitioned form

$$
\mathbf{u}=\left[u_{1}, c_{1}\left|u_{2}, c_{2}\right| \cdots \mid u_{n+1}, c_{n+1}\right]^{T},
$$

where each sub-block is of dimension $2 \times 1$, and $u_{i}$ and $c_{i}$ represent the invasive cell and ECM densities at the $i$ th node, respectively.

The nonlinear function $\mathbf{F}(\mathbf{u})$ has as its coordinates the discrete analogues (5-6), of the coupled partial differential equations (1-2):

$$
\mathbf{F}(\mathbf{u})=\left[F_{u_{1}}(\mathbf{u}), F_{c_{1}}(\mathbf{u})\left|F_{u_{2}}(\mathbf{u}), F_{c_{2}}(\mathbf{u})\right| \cdots \mid F_{u_{n+1}}(\mathbf{u}), F_{c_{n+1}}(\mathbf{u})\right]^{T} .
$$




\subsection{Nonlinear solution method}

Given the system of nonlinear discrete equations (10) we employ an inexact Newton technique [3] at each time step to solve simultaneously for the invasive cell and ECM distributions in space. We solve the system of equations in a coupled manner, such that the Jacobian matrix is block tridiagonal with $2 \times 2$ sub-blocks. Defining the vector $\mathbf{u}^{k}$ to be the $k$ th approximation to the solution vector, the linearised system $\mathbf{J}\left(\mathbf{u}^{k}\right) \delta \mathbf{u}^{k}=-\mathbf{F}\left(\mathbf{u}^{k}\right)$ is then solved for the Newton correction $\delta \mathbf{u}^{k}$ using a block implementation of the tridiagonal matrix algorithm. Here $\mathbf{J}\left(\mathbf{u}^{k}\right)$ is a numerical approximation to the Jacobian matrix computed using finite difference approximations for the derivatives [3]. The solution approximation is then updated using $\mathbf{u}^{k+1}=\mathbf{u}^{k}+\delta \mathbf{u}^{k}$ and the nonlinear iterations are continued until the convergence criterion, $\left\|\mathbf{F}\left(\mathbf{u}^{k+1}\right)\right\|<$ tol $\approx 10^{-7}$, is satisfied. The results of the simulations carried out using these computational strategies are presented in the following section.

\section{$3 \quad$ Results and comparisons}

In order to gauge the effectiveness of the control volume scheme proposed in the previous section, we now present plots resulting from numerical simulations using both the CV scheme and the NAG routine D03PCF.

The routine D03PCF integrates a system of first order, nonlinear parabolic PDEs in one spatial dimension [6]. Central, three point finite differences are used for the spatial discretisation and the method of lines is used to reduce the PDEs to a system of ordinary differential equations (ODEs). These ODEs are then solved using a backward differentiation formula. In Figure 1 we present numerical results for equations (1) and (2) using routine D03PCF.

Figure 1(a) shows the invasive cell profiles at various times using 40 spatial discretisation points. Clearly visible are vast oscillations in the solution 

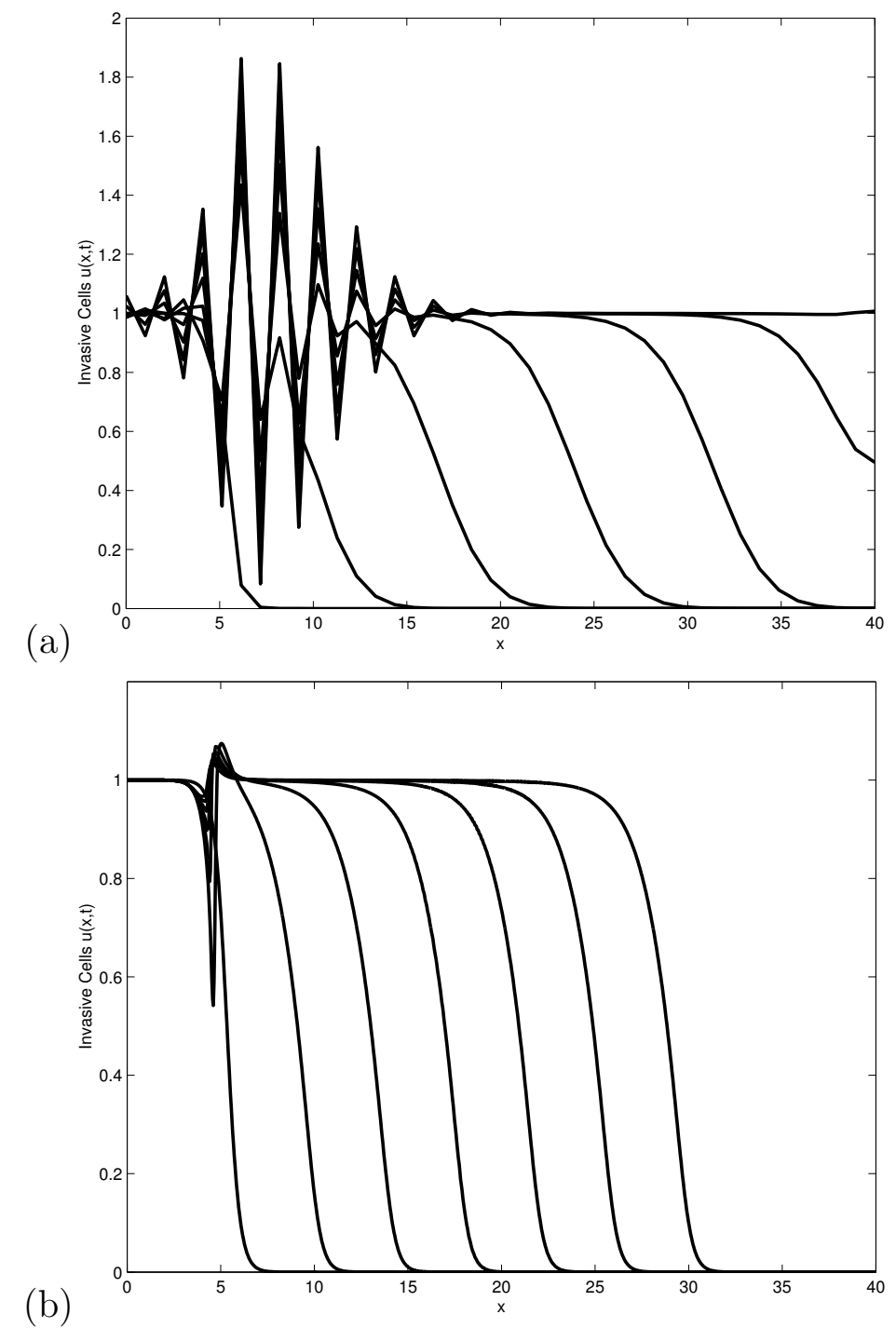

FigURE 1: NAG D03PCF results showing $u(x, t)$ for $t=0,5, \ldots, 30:$ (a) $n=$ 40 and (b) $n=1000$ spatial points; $\delta t=0.5$. 
values across almost half of the domain. These oscillation persist throughout the integration and are not dampened as $t$ increases. Furthermore, when compared with analytical results regarding the speed of travelling waves resulting from the model equations $[4,9]$, we note that the speed of propagation of the invasive cell front is considerably exaggerated. Hence, we conclude that the NAG routine is not perfectly suited to this type of problem, specifically with a coarse mesh, as illustrated by the excessive numerical diffusion and oscillatory behaviour of the solution profiles.

In Figure 1(b), we exhibit the numerical results taken from a NAG simulation using 1000 spatial discretisation points where more realistic results are evident. However, close observation reveals oscillations in the solution profiles, though here they are confined to a narrow spatial band corresponding to the area where the initial conditions have a nonzero gradient. A positive result of the mesh refinement is that the excessive numerical diffusion present in the solutions using $n=40$ spatial points is substantially decreased. Comparing the depth of migration after $t=30$ shows that for $n=40$, the front of invasive cells reaches a point past $x=40$ (the domain length), while for $n=1000$, the front has reached only as far as $x \approx 30$. This compares well with the travelling wave analytical results of $[4,9]$ and the results are not influenced by further increases in the value of $n$. As such, we will use this "fine-grid" result as a rough benchmark for our numerical solutions using the proposed control volume scheme.

In Figure 2 see that even for coarse meshes $(n=40$ and $n=50)$, the control volume scheme produces accurate results when compared with the "fine-grid" benchmark NAG solutions $(n=1000)$. Note that in both plots, the positions of the invasive cell fronts compare well with those in the benchmark plot (Figure 1(b)). The oscillations near the left boundary still appear though to a lesser extent when compared with those in the benchmark solution. Small oscillations in the invasive cell profiles will always be present at the location in question due to the choice of initial conditions [4].

Figure 3(a) shows solutions using the upstream spatial weighting method 
(a)

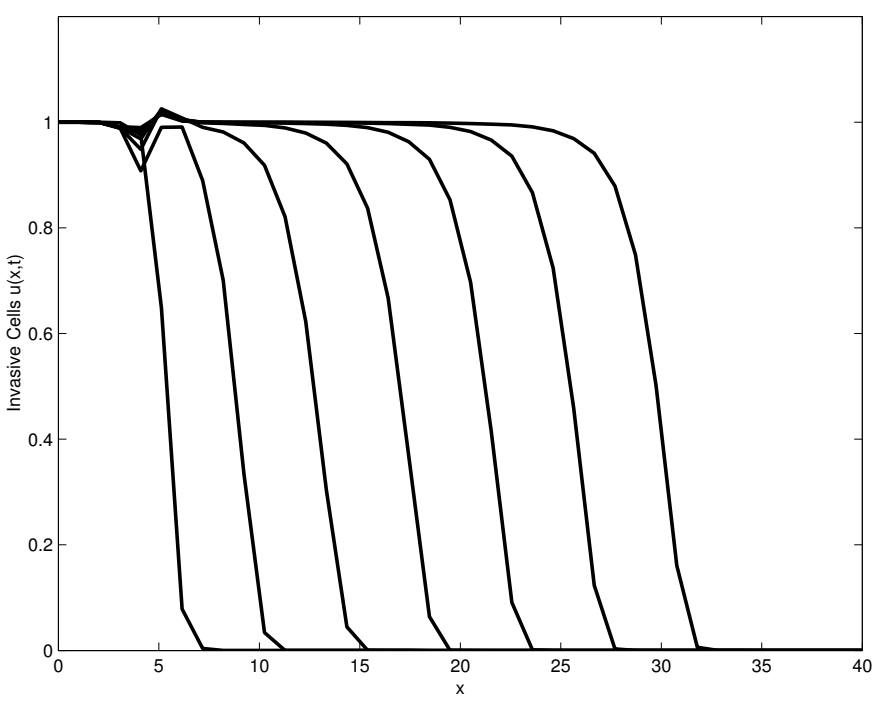

(b)

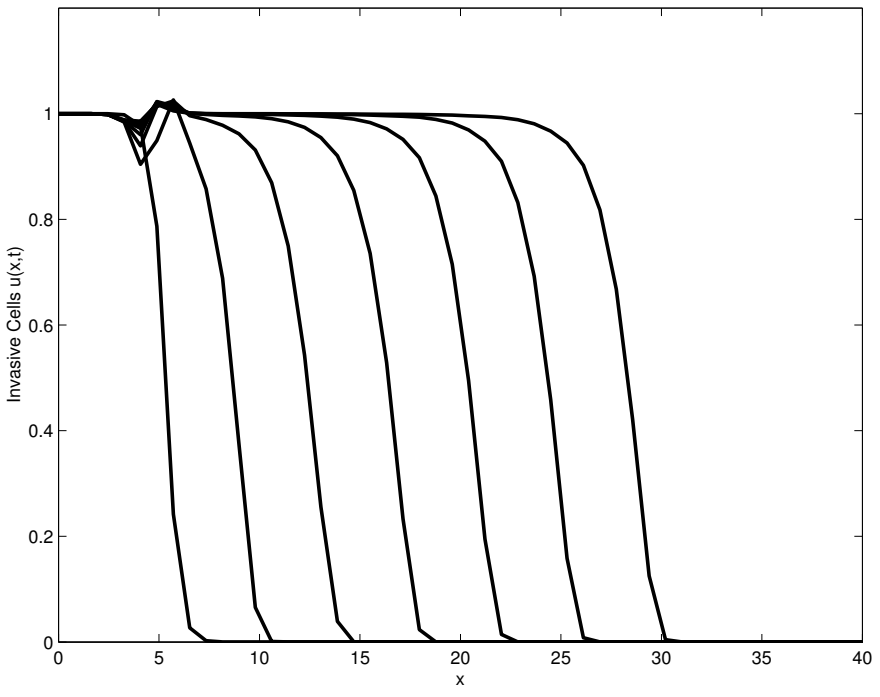

Figure 2: CV results showing $u(x, t)$ for $t=0,5, \ldots, 30$ : (a) $n=40$ and (b) $n=50$ spatial points; $\delta t_{\max }=0.5$, implicit temporal weighting, van Leer flux limiting. 
(a)

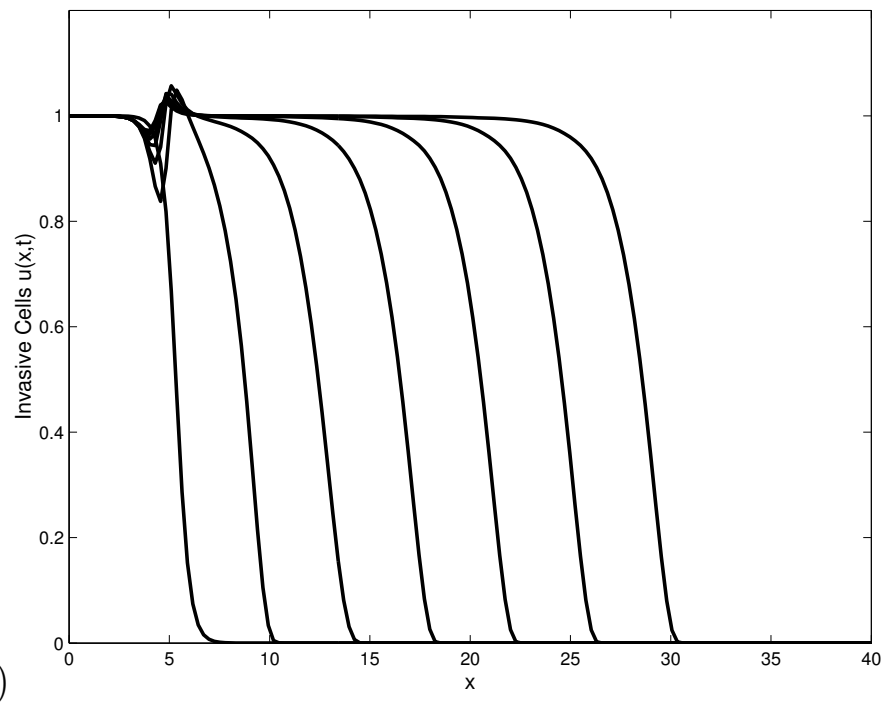

(b)

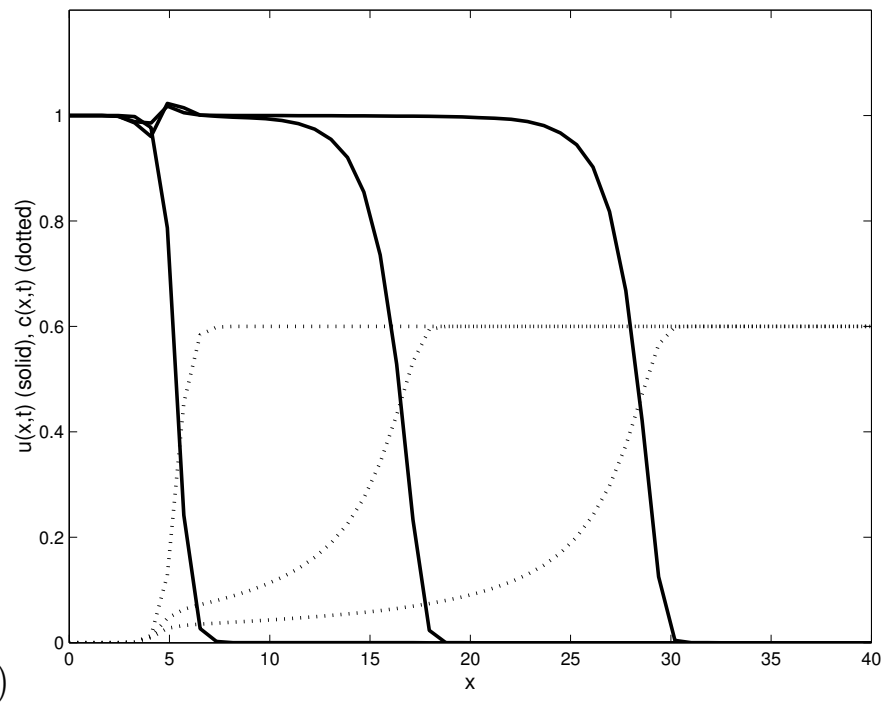

Figure 3: CV results showing: left $-u(x, t)$ for $t=0,5, \ldots, 30$, and right - $u(x, t)$ (solid) and $c(x, t)$ (dotted) for $t=0,15,30$ : (a) $n=150$ and (b) $n=50$ spatial points, $\delta t_{\max }=0.5$, implicit temporal weighting, upstream spatial weighting (a) and van Leer flux limiting (b). 
with implicit temporal weighting. Note the similarity to the coarse-mesh solutions of Figure 2, in terms of profile shape and front position. This method, though stable, has the disadvantage of requiring a vast increase in computational overheads in order to reduce numerical diffusion. Here we must have three times as many mesh points to produce similar results to those of the CV scheme with the van Leer flux limiter. Figure 3(b) uses the same specifications as Figure 2 and demonstrates the process of cell migration and ECM degradation over three time values, $t=0,15,30$ to provide a full example of the model solution.

The use of fully implicit temporal weighting with the van Leer flux limiter is found to be the best combination of spatial and temporal schemes. As expected, the use of upstream spatial weighting $(\sigma=0)$ with both fully implicit and Crank-Nicolson temporal weighting schemes results in excessive numerical diffusion - this is similar to the problem discussed above with reference to the NAG solutions in Figure 1. With mesh refinement this problem was overcome to an extent, though this did lead to unacceptable computation times. Similarly, using spatial averaging (with either temporal scheme) to approximate $u$ at the $\mathrm{CV}$ faces resulted in excessive oscillatory behaviour in the solutions due to stability constraints on the mesh size and time-step value. Finally, Crank-Nicolson temporal weighting coupled with the flux limiter produces some nonphysical results.

\section{Conclusions}

We have presented a control volume solution strategy for a nonlinear, coupled system of PDEs proposed to describe the process of tumour cell invasion. When compared with a "fine-mesh" benchmark solution using NAG routine $\mathrm{D} 03 \mathrm{PCF}$, the control volume method proves effective in producing accurate solutions as measured by the position of the front of invasive cell profiles over time. We calculated solutions using coarse meshes and the van Leer flux 
limiter, which display reduced numerical diffusion and reduced oscillatory behaviour compared with the NAG solutions.

More complicated cell migration models are constantly being proposed. These models often contain complicated flux terms and nonlinearities that make them completely unsuited to the standard "black box" solvers such as those embedded in NAG. Schemes such as the control volume method discussed here are applicable and easily adapted in cases such as these.

Acknowledgements: The authors acknowledge the QUT School of Mathematical Sciences for conference funding. The first author acknowledges QUT for a postgraduate research award.

\section{References}

[1] B. Alberts, D. Bray, J. Lewis, M. Raff, K. Roberts and J. D. Watson. The Molecular Biology of the Cell. Garland: New York, 1989. C892

[2] A. R. A. Anderson, M. A. J. Chaplain, E. L. Newman, R. J. C. Steele and A. M. Thompson. Mathematical Modelling of Tumour Invasion and Metastasis. Journal of Theoretical Medicine, 2:129-154, 2000. C893

[3] R. L. Burden and J. D. Faires. Numerical Analysis (sixth edition). Brooks/Cole Publishing Company, 1997. C897

[4] Ben Marchant. Modelling Cell Invasion. PhD Thesis. Oxford University, 1999. C893, C899

[5] B. P. Marchant, J. Norbury and J. A. Sherratt. Travelling Wave Solutions to a Haptotaxis-dominated Model of Malignant Invasion. Nonlinearity, 14:1653-1671, 2001. C893 
[6] Numerical Algorithms Group. The NAG Fortran Library Manual, Mark 19. [Online] http://www.nag.co.uk/numeric/fl/manual19/html/mark19.html. C893, C897

[7] L. Olsen, J. A. Sherratt and P. K. Maini. A Mathematical Model for Fibro-Proliferative Wound Healing Disorders. Bulletin of Mathematical Biology, 58(4):787-808, 1996. C893

[8] A. J. Perumpanani and H. M. Byrne. Extracellular Matrix Concentration Exerts Selection Pressure on Invasive Cells. European Journal of Cancer, 35(8):1274-1280, 1999. C893

[9] A. J. Perumpanani, J. Norbury, J. A. Sherratt and H. M. Byrne. A Two Parameter Family of Travelling Waves with a Singular Barrier Arising from the Modelling of Matrix Mediated Malignant Invasion. Physica D, 126:145-159, 1999. C892, C893, C894, C899

[10] B. van Leer. Towards the Ultimate Conservation Difference Scheme II, Monotonicity and Conservation Combined in a Second Order Scheme. J. Comp. Phys., 14:361-370, 1974. C896 